\title{
UMA REVISÃO SOBRE APRENDIZAGEM COLABORATIVA MEDIADA POR TECNOLOGIAS
}

A review on collaborative learning mediated by technologies

Una revisión del aprendizaje colaborativo basado en tecnología

\section{Leonardo de Andrade Carneiro ${ }^{* 1}$, Leandro Guimarães Garcia ${ }^{2}$, Gentil}

\section{Veloso Barbosa ${ }^{3}$}

${ }^{1}$ Doutorando em Desenvolvimento Regional. Programa de Pós-graduação em Desenvolvimento Regional da Universidade Federal do Estado do Tocantins. Palmas, Brasil.

${ }^{2}$ Doutor em Ciências Biológica. Docente Adjunto da Universidade Federal do Estado do Tocantins. Palmas, Brasil.

${ }^{3}$ Doutor em Engenharia de Sistemas e Computação. Docente Adjunto da Universidade Federal do Estado do

Tocantins. Palmas, Brasil.

* Correspondência: Departamento de PPGMCS, Universidade Federal do Tocantins, Brasil, Av. NS 15, 109 Norte, Palmas, Tocantins, Brasil. CEP:77.010-090.e-mail artigo@artigo.com.

\section{RESUMO}

Este trabalho apresenta um estudo acerca da aprendizagem colaborativa e suas interfaces no ensino mediado pelas tecnologias. Esta pesquisa trata-se de uma revisão que reuniu artigos e autores que tratam de conceitos sobre tecnologias digitais e a internet, instrumentos que estão inovando o modo de transmitir e coletar novos conhecimentos. Desta forma, apresenta a evolução dos processos educativos, sendo do modelo tradicional para a aprendizagem colaborativa, Aprendizagem colaborativa, comunidades virtuais de aprendizagem e redes sociais educativas, aprendizagem ubíqua. Como resultado, evidencia-se que esses métodos envolvem a interação social, que motiva os participantes a compreender os processos de colaboração e comunicação através da troca de conhecimentos utilizando diversos recursos tecnológicos.

Palavras-chave: Aprendizagem colaborativa; ensino mediado por tecnologias; interação social.

\section{ABSTRACT}

This paper presents a study about collaborative learning and its interfaces in technology-mediated teaching. This research is a review that brought together articles and authors dealing with concepts about digital technologies and the internet, instruments that are innovating the way of transmitting and collecting new knowledge. In this way, it presents the evolution of the educational processes, being of the traditional model for collaborative learning, Collaborative Learning, Virtual Learning Communities and Educational Social Networks, Ubiquitous Learning. As a result, it is evident that these methods involve social interaction, which motivates participants to understand the processes of collaboration and communication through the exchange of knowledge using various technological resources.

Keywords: Collaborative learning; technology-mediated teaching; social interaction.

\section{RESUMEN}

Este artículo presenta un estudio sobre el aprendizaje colaborativo y sus interfaces en la enseñanza mediada por la tecnología. Esta investigación es una revisión que reunió a artículos y autores que tratan conceptos sobre tecnologías digitales e Internet, instrumentos que están innovando la forma de transmitir y recopilar nuevos conocimientos. De esta manera, presenta la evolución de los procesos educativos, siendo el modelo tradicional de 
aprendizaje colaborativo, aprendizaje colaborativo, comunidades virtuales de aprendizaje y redes sociales educativas, aprendizaje ubicuo. Como resultado, es evidente que estos métodos implican la interacción social, lo que motiva a los participantes a comprender los procesos de colaboración y comunicación a través del intercambio de conocimientos utilizando diversos recursos tecnológicos.

Descriptores: Aprendizaje colaborativo; enseñanza mediada por la tecnología; interacción social

\section{INTRODUÇÃO}

As tecnologias moldam os seres humanos, e os seres humanos moldam a tecnologia, e essa construção do conhecimento é adquirida não apenas pelo acesso à informação, mas pela interação entre alunos e professores (BORBA et al., 2018).

A aprendizagem mediada por tecnologia tornou-se um tópico que vem ocupando espaço nas discussões. Essas abordagens costumam apresentar tecnologias de comunicação como ferramentas que inovam práticas educacionais, pois permitem maior flexibilidade nos métodos de aprendizagem (CARNEIRO et al., 2019).

Essas abordagens costumam apresentar as tecnologias de comunicação como instrumentos que inovam as práticas educacionais, pois permitem mais flexibilidade nos métodos formativos. Neste sentido, as inovações tecnológicas e seu uso para fins científicos, tecnológicos, culturais, sociais e educacionais foram determinantes para aproximar os benefícios da tecnologia às necessidades de qualificação (SILVA e GOMES, 2015).

Nesse sentido, a internet e sua popularização para a sociedade através das tecnologias digitais norteou o surgimento de novas estruturas, metodologias e abordagens no ensino em todos os níveis educacionais. Segundo Gannon-Leary e Fontainha (2007), a internet oferece interação com um centro universalmente acessível, democrático e interativo de comunicações e recursos rápidos e de baixo custo que conectam pessoas. E essa tecnologia da informação criou novas oportunidades para a educação. Portanto, a evolução tecnológica desempenha papel fundamental no desenvolvimento educacional, fornecendo mecanismos para evolução do processo de ensino e aprendizado de maneira a atender as demandas sociais.

Estas experiências, de ensino mediado por tecnologia, podem fornecer o aprendizado certo no momento certo e no lugar certo para cada indivíduo, não apenas experiências no trabalho, mas em escolas, transporte público, em casa (SÁNCHEZ-GÓMEZ, et al., 2017).

Os sistemas educacionais possibilitam a autoaprendizagem, com a mediação de recursos didáticos sistematicamente organizados, apresentados em diferentes suportes de informação utilizados isoladamente ou combinados, e veiculados na rede mundial de computadores (PEREIRA e ARAÚJO, 2011).

Nesse capítulo traremos breves discussões sobre redes sociais educativas, aprendizagem ubíqua e comunidades de aprendizagem permeadas pelas críticas à resistência dos modelos tradicionais em acompanhar as transformações que as tecnologias digitais propiciaram em meio século.

\section{DO MODELO TRADICIONAL PARA A APRENDIZAGEM COLABORATIVA}

Paulo Freire (1975, p. 33) nos ensinou que "O papel do educador não é o de "encher" o educando de "conhecimento", de ordem técnica ou não, mas de proporcionar, através da relação dialógica educadoreducando, educando-educador, a organização de um pensamento correto em ambos 
O que Paulo Freire chamou de relação dialógica pressupõe que o processo formativo deva investir tanto na cognição, quanto na afetividade e nos valores que transitam entre os atores do processo da aprendizagem.

Dessa forma, o professor precisa de capacidade de diálogo e criatividade, uma vez que se trata de uma estratégia bem mais complexa que leituras de textos de livros didáticos. Consideramos que assim se potencializa o fazer docente, e o educador "de mero retransmissor de saberes se torna formulador de problemas, provocando, coordenando equipes, sistematizando experiências, a memória da instituição ao longo da vida, tornando possível o diálogo entre as gerações" (BARBERO, 1996, s\p).

Salienta-se que as tecnologias desenvolvem novas formas de ensinar e aprender, resultando assim em mudanças procedimentais de aprendizagem que tendem a evoluir para uma abordagem colaborativa de e-learning.

Desse modo, a incorporação de novas práticas e softwares para solução de problemas usando tecnologias disponíveis podem ajudar a enriquecer o ensino e a experiência de aprendizado de colaboração on-line, usando ferramentas de tecnologia e fortalecendo assim a aprendizagem colaborativa (KASINATHAN et al., 2014; DOMINIC e HINA, 2016). Essa interação social aplicada na educação melhora as habilidades dos discentes, mediante grupos interdisciplinares que buscam alcançar objetivos comuns (TAWILEH, 2016).

"As teorias de aprendizagem são utilizadas na criação de ambientes instrucionais”. Elas foram criadas antes da era das tecnologias educacionais. Sendo que essa tecnologia trouxe avanços em todos os ramos da sociedade industrial, e modificou as estruturas de comunicação, transformando o conhecimento e a maneira e aprender e ensinar (MECHLOVA e MALCIK, 2012).
Na perspectiva de Mechlova e Malcik (2012) a tecnologia de informação e comunicação, possui quatro teorias de aprendizagem que foram utilizadas na criação de ambientes de ensino: "behaviorismo, cognitivismo, construtivismo e conectivismo".

Nesse sentido, descreveremos sobre o conectivismo, que é um sistema teórico que busca dominar a aprendizagem por meio tecnológico. Utilizando-se de ferramentas digitais "navegadores da Web, mecanismos de pesquisa, wikis, fóruns de discussão on-line e redes sociais" ele foi implementado como uma "teoria de aprendizagem baseada na premissa de que o conhecimento existe no mundo e não na cabeça de um indivíduo" (MECHLOVA e MALCIK, 2012).

Mechlova e Malcik (2012) descreve sete princípios do conectivismo:

Aprendizagem e conhecimento repousam na diversidade de opiniões; A aprendizagem é um processo de conexão de nós especializados ou fontes de informação; $\mathrm{O}$ aprendizado pode residir em aparelhos não humanos; A capacidade de saber mais é mais crítica do que é conhecida atualmente; Nutrir e manter conexões é necessário para facilitar o aprendizado contínuo; A capacidade de ver conexões entre campos, ideias e conceitos é uma habilidade essencial; Moeda (conhecimento preciso e atualizado) é a intenção de todas as atividades de aprendizagem conectivistas (MECHLOVA ; MALCIK, 2012).

Portanto, princípios que estabelecem critérios e definições sobre formas de saberes, a capacidade de manter-se conectado e desenvolvendo habilidades teóricas e práticas de aprendizagem, onde os saberes são distribuídos através de ferramentas digitais.

Portanto, fornecendo novas perspectivas sobre as "habilidades de aprendizado e tarefas necessárias" para que os alunos desenvolvam suas capacidades na era tecnológica (MECHLOVA e MALCIK, 2012), procurando desenvolver a aprendizagem complexa em um mundo digital social em rápida mudança (SONG, 2012). 
Song (2012) afirma ainda que;

$\mathrm{O}$ aprendizado na era digital não depende mais da aquisição, armazenamento e recuperação de conhecimento individual; em vez disso, ele depende da aprendizagem conectada que ocorre por meio da interação com várias fontes de conhecimento (incluindo a Internet e sistemas de gerenciamento de aprendizado) e participação em comunidades de interesse comum, redes sociais e tarefas de grupo (SONG, 2012, p. 423).

Dessa forma podemos afirmar que não bastam mais novos arremedos no velho tecido acadêmico. Não está mais preso aos métodos tradicionais "face-aface", os discentes devem desenvolver capacidades de analisar as diversas fontes de saberes. Os professores tradicionais precisam desenvolver novos saberes e implementar atualização profissional em suas atividades, além de vencerem a resistência das instituições em assumir as mudanças profundas e adequadas à nova realidade.

É um importante desafio superar os modelos pedagógicos centralizados no professor ou em redes hierarquizadas que não permitem que as informações e as interações fluam em todos os circuitos da comunicação educacional

\section{APRENDIZAGEM COLABORATIVA}

Segundo Schimitz e Foelsing (2018), aprendizagem colaborativa refere-se a processos de interação social em que os alunos trabalham ativamente em conjunto com objetivos de aprendizagem compartilhada e participam de um processo de ensino-aprendizagem. Portanto, na premissa de que o conhecimento é social e construído a partir de esforços colaborativos para aprender, entender e resolver problemas (SONG, 2012).

Song (2012) define ainda que em um ambiente de aprendizado colaborativo, os alunos não são mais "receptores passivos, mas exploradores ativos em seu processo de aquisição de conhecimento", participativos, autogerenciados e tendem a compartilhar informações com seus pares.

Podemos afirmar que o principal objetivo da aprendizagem colaborativa é a participação ativa dos membros. Isso é possível, porque a interdisciplinaridade dos alunos pode fomentar novas descobertas a partir dos feedbacks e apoios. Essa dinâmica aperfeiçoa as práticas de aprendizagem.

Para Rezagholilalani e Ibrahim (2017), a aprendizagem colaborativa é definida como uma abordagem educacional. Ela enfatiza os esforços ativos e coletivos de participação e interação por parte dos alunos e do instrutor principalmente pela comunicação via internet dentro do novo paradigma educacional. O foco é aprendizagem interativa e centrada no aluno tendo em vista que os trabalhos são normalmente feitos em grupos que interagem para atingir um mesmo objetivo.

Para Adedoyin et al. (2018); Alvarado et al. (2016); Webb et al. (2018), a aprendizagem colaborativa refere-se a tarefas que exigem esforços intelectuais ativos entre os grupos e em conjunto com objetivos de aprendizagem compartilhados. Portanto, implica em uma filosofia pessoal, não apenas em uma técnica de sala de aula. Carneiro e Barbosa (2018) esclarecem que "a interação social, além de fonte para o compartilhamento de informações, torna-se fonte de colaboração de conhecimento, ocorrendo desta forma, conflitos intelectuais" (p.?).

Para Lévy (1999), a estrutura física, formada pelos dispositivos digitais, móveis e redes de comunicação, possibilitou uma grande quantidade de informações que, manipuladas pelo homem, possibilitaram a criação do ciberespaço. Esse ambiente suporta tecnologias intelectuais que possibilitam o desenvolvimento das funções cognitivas das pessoas.

Essas tecnologias constituem processos que se convertem em informações que podem ser compartilhadas em grande escala. O pensamento de 
Lévy nos remete aos benefícios do ensino mediado pelas tecnologias. Esse formato tem potencial para o desenvolvimento do conhecimento de forma colaborativa entre seus membros, viabilizado pelos recursos tecnológicos e possibilitado pelas redes digitais.

Esses mecanismos imprimem outra dinâmica no processo de ensinar e aprender que vai desde a flexibilização do tempo até o espaço. Nesse caso, não se trata de autoaprendizagem, mas da aprendizagem mediada por uma comunidade que motiva, esclarece, orienta, embora não haja a sistematicidade que os cursos tradicionais preconizam.

É inquestionável que o uso de dispositivos móveis tende a potencializar novas formas de aprendizagem, gerando reflexões no ensino por meio do compartilhamento de ideias através da aprendizagem colaborativa. Essas informações estão disponíveis em dispositivos que cabem na palma da mão como os smartphones e tablets, entre outros presentes em todos os lugares.

O desenvolvimento dessas tecnologias facilita o compartilhamento de espaços de colaboração e produção, distribuição e agregação de informações em ambientes on-line de aprendizagem (RENÓ, et al., 2018).

\section{COMUNIDADES}

APRENDIZAGEM EDUCATIVAS

Comunidade virtual de aprendizagem é uma rede de indivíduos que compartilham experiências e metodologias de interesse. Como afirma GannonLeary e Fontainha (2007), cada participante na comunidade contribui para o desenvolvimento do conhecimento por meio de educação e desenvolvimento tecnológico permitindo maior comunicação e interatividade entre eles, incorporando, dessa forma, novos modelos pedagógicos colaborativos de forma síncrona e assíncrona, dando acesso às comunidades geograficamente isoladas e compartilhando informações em locais distintos.

A busca por conhecimento faz com que novos membros sejam incluídos nessas comunidades de conhecimento. Sendo assim, o aprendizado dos discentes pode ser aprimorado por meio do envolvimento com outras pessoas. A conscientização tecnológica, a motivação e o comportamento de mudança de aprendizes são pré-requisitos para implementações bem-sucedidas dessas modalidades de ensino por meio de comunidades virtuais (BHUASIRI, et al., 2012).

$\mathrm{O}$ aprendizado que se desenrola nessas “comunidades é colaborativo, no qual o conhecimento colaborativo da comunidade é maior do que qualquer conhecimento individual", e as pessoas "aprendem melhor através da interação social" (GANNONLEARY e FONTAINHA, 2007).

As comunidades de aprendizagem fornecem benefícios para as partes interessadas, e as vantagens dessa modalidade incluem maior acessibilidade à informação, padronização de conteúdo, responsabilidade, interatividade, confiança e maior conveniência (BHUASIRI, et al., 2012). A tecnologia da informação está aumentando sua importância nessas comunidades, possibilitando uma maior integração dos discentes, aperfeiçoando métodos e aprimorando técnicas necessárias para os sucessos dessas comunidades.

A aprendizagem colaborativa constitui-se uma oportunidade para integrar as tecnologias, sendo que as ferramentas de aprendizagem possibilitam e potencializam a construção dos conhecimentos através de um suporte colaborativo.

Essas comunidades usam a rede social existente em uma rede virtual (tecnologia) para a troca de informações de interesse comum. Portanto, a comunidade emerge da tipologia e interações de 
colaboração que ocorrem entre indivíduos, constituindo-se no suporte para o desenvolvimento do compartilhamento de interesses e objetivos na colaboração do conhecimento (DIAS, 2004). Sendo assim, os principais objetivos das comunidades de aprendizagem é a participação ativa dos membros fomentando novas descobertas. Elas permitem a interação entre os indivíduos (aprendizagem colaborativa) e o objetivo é a troca permanente de conhecimento por meio de atividades de aprendizagem e aperfeiçoamento.

Segundo Campanella e Impedovo (2015) e (Kasinathan et al. (2014), a sociedade a cada dia utiliza as mídias sociais no ensino usando dispositivos móveis para melhorar a experiência de aprendizado de forma colaborativa. As redes sociais educativas fornecem métodos colaborativos, imediatos e relevantes para obter conhecimento compartilhado. Esse é um dado positivo do uso de redes sociais (LEE; LEE, 2018). Nesse sentido, elas podem promover o aprendizado compartilhado uma vez que vários usuários com objetivos semelhantes visitam e visualizam as postagens e os conteúdos das pessoas.

Contudo, adicionar essa colaboração em rede para a sala de aula é insuficiente. As ferramentas de aprendizagem devem ser incluídas de maneiras estratégicas promovendo um novo pensar crítico para avaliar a adoção, a implantação e o uso efetivo para colaboração (ARMFIELD et al., 2014).

Uma rede social educativa é indicada para grupos de sujeitos aprendentes com interesses comuns. Nela o compartilhamento de conhecimentos se desenvolve num ambiente adequado para a aprendizagem colaborativa e ela ocorre devido a participação dos integrantes do grupo, podendo ser discentes ou docentes. Esse sistema favorece e depende da participação social, porque é fruto de uma composição social e ocorre onde há a interação e a colaboração das pessoas (ABREU et al., 2011). Long at al. (2019) afirmam que a rede social educativa é um método altamente social, onde ocorre interação e compartilhamento de conhecimentos com outros de forma sinérgica, ou seja, uma rede de compartilhamento de conhecimento.

Abreu et al. (2011) afirmam que:

Um ambiente de aprendizagem colaborativo deve ser rico em possibilidades e propiciar o crescimento do grupo. As redes sociais são vistas como ambientes que propiciam a prática de colaboração entre os indivíduos. As redes sociais são ambientes que incentivam a construção do conhecimento de modo colaborativo, uma vez que possibilitam um constante intercâmbio de informações entre os seus membros (ABREU et al., 2011, p. 1248).

Portanto as redes sociais educativas são um mecanismo que integra as pessoas objetivando construírem o conhecimento e proporcionando uma aprendizagem mais eficiente e eficaz. Essa metodologia de compartilhar conhecimento transformou o espaço das salas de aula em ambientes móveis. Se a escola adota o expediente e uso da tecnologia virtual, os discentes têm a possibilidade de levar seu aprendizado de modo mais efetivo para fora da entidade escolar e o docente precisa repensar o espaço virtual e o presencial como espaços integrados (SILVA; SCHIMIGUEL, 2013).

Lee e Lee (2018) enfatizam que o uso de redes sociais pode melhorar o desempenho do aprendizado somente quando os usuários participam de grupos e compartilham seus conhecimentos e ideias, tendo em vista a interdisciplinaridade das formações dos participantes dos grupos de estudos ou discussões.

Para Carneiro e Barbosa (2018, p. 54), "as ferramentas de aprendizagem devem combinar teoria e prática, espaço e tempo, definindo estratégias como fatores essenciais para uma aprendizagem mais autônoma e dinâmica, favorecendo múltiplos caminhos de ensino". Nesse sentido, as redes sociais educativas podem motivar os participantes a produzirem e desenvolverem resultados melhores. 
Para isso, é importante a implementação de ferramentas que já estão em uso, a inovação das oportunidades de conhecimento e também aperfeiçoar os métodos e técnicas. Dentro desse perfil, o professor deve atuar como moderador para que os alunos desenvolvam seus saberes em interação com o outro e não apenas o adquiram como um papel em branco (BULGRAEN, 2010).

As ferramentas de rede social virtual podem facilitar o compartilhamento de conhecimento, incentivando os membros a participar do processo de aprendizagem, no desenvolvimento das habilidades de comunicação e informação (AL-SAMARRAIE E SAEED, 2018). O uso dessa mídia pode ser essencial para melhorar a eficiência de aprendizagem quando os alunos têm interesse e estão automotivados para usálas para aprender (LEE E LEE, 2018). Portanto o aprendizado colaborativo associado às redes sociais poderá favorecer resultados positivos, pois as redes sociais virtuais, além de promover a interatividade entre os indivíduos, permitem que cada integrante exponha suas ideias, compartilhe conhecimento e até mesmo emoções.

Vivenciamos um tempo marcado pela interatividade, mobilidade e ubiquidade em nossas vidas influenciado por nossos estilos de vida (SILVA e FALAVIGNA, 2018).

Santaella (2010, p. 17) define a educação ubíqua como processos de aprendizagens abertos, espontâneos, assistemáticos e com acesso contínuo à informação e a qualquer hora. Desta forma, a aprendizagem ubíqua baseia-se na aprendizagem interativa por meio das tecnologias digitais que possibilitam aos estudantes o acesso às informações e mediação com o uso de qualquer dispositivo que acesse as redes virtuais (VIRTANEN, et al., 2016). Nesse sentido, apenas reforça um fenômeno já existente, afinal sempre aprendemos em qualquer lugar.
Para Montoya (2009, apud Piaget, 1972) a aprendizagem é “acomodação á experiencia dos esquemas de assimilação", portanto, "aprender é construir estrutura de assimilação" e a "aprendizagem é função dos instrumentos lógicos à disposição das pessoas".

As qualidades instrucionistas de aplicativos de aprendizado móveis em dispositivos móveis podem facilitar o desenvolvimento cognitivo dos alunos no ambiente escolar. Além da aprendizagem colaborativa por meio do uso de dispositivos móveis, criando novas oportunidades educacionais. Melhorando o ambiente formal de aprendizado mais diretamente com práticas de aprendizado informal e, por sua vez, torna essas experiências educacionais mais prontamente disponíveis para posterior reflexão e discussão em sala de aula (PIMMER et al., 2016).

$$
\text { Cárdenas-Robledo e Peña-Ayala }
$$
afirmam que:

O aprendizado ubíquo aproveita o conteúdo digital, o ambiente físico, os dispositivos móveis, os componentes difundidos e a comunicação sem fio para fornecer experiências de ensinoaprendizagem aos usuários a qualquer momento, em qualquer lugar e de qualquer maneira.

A disponibilidade de informação e comunicação para a educação possibilita uma formação continuada e sem os deslocamentos que o mundo analógico exige. Dessa forma, a integração de ferramentas educacionais no ensino pode motivar a geração Z, que são estudantes sociais, móveis, globais, digitais e visuais (LAHUERTA et al., 2018). Essas tecnologias digitais fornecem oportunidades para os discentes, bem como professores na resolução de situações em tempo real com a utilização de ferramentas de aprendizagem (CLOONAN, 2018).

A aprendizagem móvel é apresentada como um conjunto de medidas que através de conversa podese ter múltiplos contextos, visando o envolvimento dos alunos nos processos educativos, promovendo desta 
forma, novas formas de aprender, fora do ambiente escolar. Portanto as tecnologias móveis e portáteis permitem que discentes acessem informações em qualquer lugar e tempo (CARNEIRO e BARBOSA, 2018 apud PIMMER et al., 2016).

Os avanços tecnológicos e a implementação de novas ferramentas digitais na aprendizagem onipresente ou ubíqua surgem como instrumento de oportunidades para aprendizagem em qualquer lugar e hora. (DOMINIC e HINA, 2016) descrevem que as ferramentas digitais e tecnologias permitem que alunos assumam a autonomia de sua aprendizagem, estabelecendo o contexto que os inspira na busca por novas formas de aprender.

A internet é essencial para o desenvolvimento cognitivo ocasionado pela modernização da sociedade com aplicações inovadoras baseadas em interações sociais (CARNEIRO e BARBOSA, 2018), contudo não é apenas tratar de mudar o conceito do que é importante, mas sim de encontrar a junção das ferramentas digitais para a aprendizagem (ESICHAIKULA et al., 2013; KU e CHEN, 2015). A colaboração é essencial no desenvolvimento educacional por meio da aprendizagem onipresente, pois proporciona novas oportunidades de discussão e reflexão sobre os saberes existentes. A aprendizagem onipresente e o uso de ambientes de aprendizagem onipresentes anunciam uma nova era nas relações de aprendizado e ensino. Esses ambientes aprimoram as experiências de aprendizado em qualquer lugar e a qualquer momento (VIRTANEN, et al., 2018).

\section{CONCLUSÃO}

A evolução das tecnologias e a difusão do conhecimento estão se tornando cada vez mais incisivos na forma como os usuários compartilham informações. Destarte, as pessoas conectadas à web podem acessar milhões de informações apenas ao clicar um botão. Sendo que as tecnologias digitais e a internet inovaram o modo de transmitir e coletar novos conhecimentos.

Sua importância reside nas necessidades de interação que proporcionam cada vez mais para a sociedade facilidades para o aprendizado, que podem ser aplicadas como uma ferramenta para o trabalho consulta e publicação de informações importantes.

Além disso, as tecnologias digitais estão formalizadas e oferecendo cada vez mais possibilidades de aprendizagem. Pode-se afirmar que, os ambientes de aprendizagem começaram a crescer e expandir à medida que essas tecnologias expandiram possibilidades de comunicação e interação.

As descobertas sugerem que a experiência de aprendizagem colaborativa é essencialmente construtiva, uma vez que, prepara discentes para as diversas atividades institucionais adquirindo saberes necessários para resolução de problemas com a participação e colaboração de outros.

Precisamos apropriarmo-nos das incertezas e transformar a educação em processos de formação permanente, pois nenhuma formação atual dá garantias que terá qualquer valor daqui a uma década.

Esse texto demonstra que a inovação das metodologias de aprendizagem ocasionadas pela disseminação da informação e as tipologias de ensinar e aprender por meio das redes sociais educativas, aprendizagem ubíqua ou onipresente e comunidades de aprendizagens, desenvolvem nos aprendizes (discentes e docentes) métodos altamente sociais, tendo em vista a interação dos envolvidos no compartilhamento de conhecimento, ideias e habilidades.

Uma aprendizagem colaborativa, "uma rede de compartilhamento de conhecimento" como afirma (LONG, et al., 2019), é onde as oportunidades para aprendizagem ocorrem em qualquer lugar e hora, como um processo de aprender e ensinar, sem limites ou local. Dessa forma, as metodologias baseiam-se na 
interativadade dos usuários ocasionada pelo acesso fácil às informações disponivéis.

Neste sentido, esses métodos envolvem a interação social, que motiva os participantes a compreender os processos de colaboração e comunicação através da troca de conhecimentos utilizando diversos recursos tecnológicos.

Diante disso, as mudanças tecnológicas estão afetando os ambientes de aprendizado e possibilitando uma melhor adaptação das instituições por novas demandas. Em consequência disso, a colaboração digital tornou-se essencial para estudantes, professores e profissionais em diversas áreas do conhecimento, desenvolvendo assim, novas fontes de aprendizado e difusão de novos saberes de forma colaborativa.

Todos os autores declararam não haver qualquer potencial conflito de interesses referente a este artigo.

\section{REFERÊNCIAS}

ABREU, João et al. Análise das práticas de colaboração e comunicação: estudo de caso utilizando a Rede Social Educativa Redu. In: Anais do Workshop de Informática na Escola. 2011. p. 12461255 .

ADEDOYIN, Adeyinka et al. Development of a Web-based University Collaborative Tool for Effective Distributed Learning. AFRICAN JOURNAL OF COMPUTING \& ICT, p. 33, 2018.

AL-SAMARRAIE, Hosam; SAEED, Noria. A systematic review of cloud computing tools for collaborative learning: Opportunities and challenges to the blended-learning environment. Computers \& Education, v. 124, p. 77-91, 2018.

ALVARADO, J. V. et al. Collaborative logical framework: An e-learning assesment tool in.LRN platform. Proceedings - 2016 11th Latin American Conference on Learning Objects and Technology, LACLO 2016. [S.1.]: [s.n.]. 2016.

ARMFIELD, D. M.; KENNEDY, K.; DUIN, A. H. Extended abstract: The roles of networked learning, collaboration, and connectedness in the classroom. IEEE International Professional Communication Conference. [S.1.]: [s.n.]. 2015.
BHUASIRI, Wannasiri et al. Critical success factors for e-learning in developing countries: A comparative analysis between ICT experts and faculty. Computers \& Education, v. 58, n. 2, p. 843-855, 2012.

BORBA, Marcelo C.; DE SOUZA CHIARI, Aparecida Santana; DE ALMEIDA, Helber Rangel Formiga Leite. Interactions in virtual learning environments: new roles for digital technology. Educational Studies in Mathematics, v. 98, n. 3, p. 269-286, 2018.

BULGRAEN, Vanessa C. O papel do professor e sua mediação nos processos de elaboração do conhecimento. Revista Conteúdo, Capivari, v. 1, n. 4, p. $30-38,2010$.

CAMPANELLA, P.; IMPEDOVO, S. Innovative Methods for the E-learning Recommendation. In: 2015 Fifth International Conference on Digital Information Processing and Communications (ICDIPC). IEEE, 2015. p. 312-317.

CÁRDENAS-ROBLEDO, Leonor Adriana; PEÑAAYALA, Alejandro. Ubiquitous learning: A systematic review. Telematics and Informatics, v. 35, n. 5, p. 1097-1132, 2018.

CARNEIRO, Leonardo de Andrade; PRATA, D. N.; MOREIRA, P. L.; BARBOSA, G. V.. Collaborative Learning in the Military Police of Tocantins: perspective without frontier. INTERNATIONAL JOURNAL OF ADVANCED ENGINEERING RESEARCH AND SCIENCE, v. 6, p. 39-45, 2019.

CARNEIRO, Leonardo de Andrade; BARBOSA, Gentil Veloso. Uma análise crítica sobre aprendizagem: colaborativa e Móvel ubíqua. Humanidades \& Inovação, [S.1.], v. 5, n. 11, p. 50-54, dec. 2018. ISSN 2358-8322.

CLOONAN, A. Collaborative teacher research: integrating professional learning and university study. The Australian Educational Researcher, 2018. ISSN 10.1007/s13384-018-0290-y.

DIAS, P. Aprendizagem colaborativa e comunidades de inovação. In: ALMEIDA, M. E. B. D.; DIAS, P.; SILVA, B. D. D. Cenários educativos de inovação na sociedade digital. São Paulo: Loyola, 2013. p. 1320.

DIAS, Paulo. Comunidades de aprendizagem e formação on-line. Nov@ Formação: revista semestral sobre formação a distância \& e-Learning, p. 14-17, 2004. 
DOMINIC, D. D.; HINA, S. Engaging university students in hands on learning practices and social media collaboration. 2016 3rd International Conference on Computer and Information Sciences, ICCOINS 2016 - Proceedings. [S.1.]: [s.n.]. 2016.

ESICHAIKUL, V. et al. Development and evaluation of wiki collaboration space for e-Learning. Journal of Enterprise Information Management, 2013. ISSN 10.1108/JEIM-07-2013-0045.

FREIRE, Paulo. Pedagogia do oprimido. $17^{\text {a }}$. Ed. Rio de Janeiro: Paz e Terra, v. 3, p. 343-348, 1987.

GANNON-LEARY, Pat; FONTAINHA, Elsa. Communities of Practice and virtual learning communities: benefits, barriers and success factors. Barriers and Success Factors. eLearning Papers, n. 5, 2007.

KASINATHAN, Vinothini; ABDUL RAHMAN, N.; CHE ABDUL RANI, M. Approaching Digital Natives with QR Code Technology in Edutainment. International Journal of Education and Research, v. 2, n. 4, p. 169-178, 2014.

KU, D. T.; CHEN, N. L. Influence of wiki participation on transnational collaboration learning anxiety in middle school students: A case study of Google wiki. Internet Research, 2015. ISSN 10.1108/IntR-05-2014-0142.

LAHUERTA-OTERO, E.; CORDEROGUTIÉRREZ, R.; IZQUIERDO-ÁLVAREZ, V. Like it or die. Proceedings of the Sixth International Conference on Technological Ecosystems for Enhancing Multiculturality - TEEM'18. [S.1.]: [s.n.]. 2018.

LAHUERTA-OTERO, E.; CORDEROGUTIÉRREZ, R.; IZQUIERDO-ÁLVAREZ, V. Like it or die. Proceedings of the Sixth International Conference on Technological Ecosystems for Enhancing Multiculturality - TEEM'18. [S.1.]: [s.n.]. 2018.

LEE, HyunKyung; LEE, MyungGeun. Social learning constructs and employee learning performance in informal Web-based learning environments. Journal of Workplace Learning, v. 30, n. 6, p. 394-414, 2018.

LÉVY, Pierre. A inteligência coletiva: por uma antropologia do ciberespaço. São Paulo: Loyola, 1994. _. As tecnologias da inteligência, 1999.

LONG, Janet C. et al. Building a learning community of Australian clinical genomics: a social network study of the Australian Genomic Health Alliance. BMC medicine, v. 17, n. 1, p. 44, 2019.

MARTINS, J. L.; SILVA, B. Narrativas da dependência nas redes de aprendizagem online: Como os professores usam as redes de aprendizagem para promover a autonomia. Holos, p. 16-30. http://dx.doi.org/10.15628/holos.2016.4002 , 2016.

MECHLOVA, E.; MALCIK, M. ICT in changes of learning theories. In: 2012 IEEE 10th International Conference on Emerging eLearning Technologies and Applications (ICETA). IEEE, 2012. p. 253-262. MONTOYA, Adrián Oscar Dongo. Teoria da aprendizagem na obra de Jean Piaget. UNESP, 2009.

PEREIRA, A. G.; ARAUJO JUNIOR, P. D. C. F. Análises preliminares de monografias: ensino a distância na Polícia Militar do Estado de São Paulo. Thesis (São Paulo. Online), v. I, p. 41-60, 2011.

PIMMER, Christoph; MATEESCU, Magdalena; GRÖHBIEL, Urs. Mobile and ubiquitous learning in higher education settings. A systematic review of empirical studies. Computers in Human Behavior, v. 63 , p. 490-501, 2016.

RENÓ, Denis Porto; TYMOSHCHUK, Oksana; SILVA, Paula Alexandra. Redes, comunidades y cultura digital: la innovación por la desconexión. Chasqui. Revista Latinoamericana de Comunicación, n. 137, p. 191-207, 2018.

REZAGHOLILALANI, Shahla; IBRAHIM, Othman. THE EFFECTS OF COLLABORATIVE LEARNING TOOLS ON STUDENTS' PERFORMANCE. Class Project Book Chapter Innovations in Information System Series: 3 | Year: 2017 | ISBN: 978-967-019497-4.

SÁNCHEZ-GÓMEZ, Ma et al. The Impact of Wikis and Discussion Boards on Learning English as a Second Language. A Mixed Methods Research. Digital Education Review, v. 32, p. 35-59, 2017.

SANTAELLA, Lucia. Desafios da ubiquidade para a educação. Revista Ensino Superior Unicamp, v. 9, p. 19-28, 2013.

SCHMITZ, Anja P.; FOELSING, Jan. Social Collaborative Learning Environments: A Means to Reconceptualise Leadership Education for Tomorrow's Leaders and Universities? In: The Disruptive Power of Online Education: Challenges, Opportunities, Responses. Emerald Publishing Limited, 2018. p. 99-123. 
SIEMENS, G. Learning Ecology, Communities, and Networks Extending the classroom. www.elearnspace.org/Articles/learning_communities. htm: [s.n.], 2003.

SILVA, B. A Glocalização da Educação: da escrita às comunidades de aprendizagem. Actas do $5^{\circ}$ Congresso da Sociedade Portuguesa de Ciências da Educação. Porto (Portugal): [s.n.]. 2002. p. 779-788.

SILVA, B. Aprendizagem na cibercultura: um novo olhar sobre as tecnologias de informação e comunicação digital no contexto educativo ubíquo. Interfaces cientificas, Aracaju, v. 6, n. 3, p. 17-28, 2018.

SILVA, Bento Duarte da; FALAVIGNA, Gladis. Aprendizagem ubíqua na modalidade b-learning: estudo de caso do Mestrado de Tecnologia Educativa da UMinho. 2016.

SILVA, Carlos; FIALHO, Joaquim; SARAGOÇA, José. Iniciação à Análise de Redes Sociais. Casos Práticos e Procedimentos com UCINET. SILVA, 2013.

SILVA, Josney Freitas; SCHIMIGUEL, Juliano. O uso das TICS no ensino superior: a integração de diferentes tecnologias à educação estatística. Revista de Produção
SILVA, Viviane Gomes; GOMES, Maria João. Dos dispositivos móveis à aprendizagem ubíqua: da usabilidade técnica à usabilidade pedagógica. In: ACTAS DEL XIII CONGRESO INTERNACIONAL GALLEGO-PORTUGUÉS DE PSICOPEDAGOGÍA. 2015.

SONG, Jie et al. Creating effective collaborative learning in a CALL environment. In: 2012 International Symposium on Information Technologies in Medicine and Education. IEEE, 2012. p. 422-425.

TAWILEH, W. Evaluating Virtual Collaborative Learning platforms using Social Network Analysis. 2016 6th International Conference on Digital Information Processing and Communications, ICDIPC 2016. [S.1.]: [s.n.]. 2016.

VIRTANEN, M. A. et al. The Comparison of Students' Satisfaction between Ubiquitous and Web-Basedlearning Environments. Education and Information Technologies, v. 22, n. 5, p. 2565-2581, 2016. ISSN 10.1007/s10639-016-9561-2.

WEBB, S. et al. Examining the use of Web-Based Tools in Fully Online Learning Community Environments, Universal Design \& Higher Education in Transformation Congress. [S.1.]. 2018. 